related to visuospatial impairment $(\mathrm{p}=0.02)$ but not to global measures of cognition, motor severity, age or disease duration. Factor analysis confirmed a unique relationship between color discrimination, visual hallucinations and visuospatial function.

Conclusion Our results suggest that color impairments may be a specific biomarker of visual hallucinations and associated visuoperceptual deficits in evolving Lewy body disorders rather than dementia per se and thus providing insight into a shared pathophysiological substrate.

\section{TRAINING IN HEADACHE IN AUSTRALIA, NEW ZEALAND AND ASIA}

2,1 ${ }^{1}$ in Zhang ${ }^{*}{ }^{3}$ Bronwyn Jenkins, ${ }^{2,1}$ Richard Stark, ${ }^{2,1}$ Elspeth Hutton. ${ }^{1}$ Neurosciences, The Alfred Hospital, Melbourne, VIC, Australia; ${ }^{2}$ Faculty of medicine nursing and health sciences, Monash University, Melbourne, VIC, Australia; ${ }^{3}$ Neurology Department, Royal North Shore Hospital, Sydney, NSW, Australia

\subsection{6/jnnp-2019-anzan.53}

Introduction Migraine is the leading cause of age-adjusted neurological disability in Australia, but little is known about headache training in our region. We aimed to assess the quantity of teaching in headache subjects during undergraduate and postgraduate years.

Method This is a cross-sectional survey study where questionnaires were sent to 137 delegates from Australia, New Zealand and Asia, prior to the Headache Master School in Sydney in August 2018. The Main outcome measured are recalled number of hours of teaching in undergraduate year and postgraduate years in: 1) Migraine; 2) Trigeminal autonomic cephalalgias (TACs); 3) Asthma; 4) Myasthenia gravis (MG).

Results The questionnaire response rate was 73\% (100 of 137), of which 29 delegates were within 10 years of completing their undergraduate degree and 98 were neurologists. In undergraduate training, there was much greater quantity of teaching in asthma than migraine $(Z=5.007$, $\mathrm{p}<0.000$ ) despite both being high-prevalent (asthma 11\%, migraine 15-20\%) conditions. Similarly, for diseases of medium-to-low prevalence, there was less training in TACs (1/1000), compared to MG (1.2/10,000) ( $Z=6.196$, $\mathrm{p}<0.000)$. These major differences in training were also seen in postgraduate years even though overall headache teaching was greater in postgraduate than undergraduate training $(\mathrm{p}<0.000)$.

Conclusions Despite the high prevalence and morbidity of headache disorders, they receive less attention in training than conditions with similar prevalence. We propose that headache training opportunities should be improved in our region, particularly in the undergraduate course and preceptorships or fellowships in postgraduate years.

\section{2 ALCOHOL ASSOCIATED TOXIC MYELOPATHY WITH NORMAL HEPATIC FUNCTION}

Srimathy Vijayan*, Scott Davies, Wai Leong. Neurology, Royal Perth Hospital, Perth, WA, Australia

10.1136/jnnp-2019-anzan.54
Introduction Alcohol related neurological dysfunction affects the central and peripheral nervous system. We present a rare consequence of excessive alcohol consumption. A 42-year-old female consuming 2 bottles of wine daily for the preceding 5 years presented with 12 months of progressive gait disturbance. Examination revealed a spastic paraparesis with prominent dorsal column signs. Extensive work up found no alternate cause for her presentation. The final diagnosis was direct alcohol mediated toxic myelopathy. We reviewed the current literature on this rare condition.

Methods We searched PubMED/OVID databases with the terms 'alcohol AND myelopathy'.

Results There is a paucity of research on this clinical entity. Sage et al. ${ }^{1}$ described 5 well-nourished alcoholics presenting with a progressive myelopathy. As with our patient, routine laboratory tests, including nutritional screen, were normal. CT Myelogram revealed no structural cause. CSF was normal. In contrast, a myelopathic syndrome can occur in alcoholic hepatic failure, attributed to portosystemic shunting of blood, with resultant hyper-ammonaemia and demyelination of corticospinal tracts. ${ }^{2-4}$

Conclusion Alcohol associated toxic myelopathy is a rare entity, distinct from hepatic myelopathy. It is thought to result from direct toxic effects of ethanol on corticospinal and large myelinated sensory tracts. The sensory involvement is unique for this entity, which distinguishes it from hepatic myelopathy, where corticospinal involvement predominates. It is prudent to look for other potentially treatable secondary causes of myelopathy. Abstinence from alcohol results in modest improvement in symptoms. Minimal literature exists on this clinical entity and further research is required.

\section{REFERENCES}

1. Sage Jl, et al. Alcoholic myelopathy without substantial liver disease. A syndrome of progressive dorsal and lateral column dysfunction. Arch Neurol. 1984 Sep;41 (9):999-1001.

2. Premkumar $\mathrm{M}$, et al. Hepatic Myelopathy in a Patient with Decompensated Alcoholic Cirrhosis and Portal Colopathy. Volume 2012, Article ID 735906, 4 pages.

3. Koo JE, et al. Hepatic myelopathy as a presenting neurological complication in patients with cirrhosis and spontaneous splenorenal shunt. Korean J Hepatol. 2008 Mar;14(1):89-96.

4. Kori $\mathrm{P}$, et al. Hepatic myelopathy: an unusual neurological complication of chronic liver disease presenting as quadriparesis. BMJ Case Rep. 2013; 2013: bcr2013009078

\section{HEADACHE WITH PERIORBITAL ECCHYMOSIS; A RARE CLINICAL ENTITY}

Srimathy Vijayan*, Carolyn Orr, Catherine Franconi. Neurology, Royal Perth Hospital, Perth, WA, Australia

\subsection{6/jnnp-2019-anzan.55}

Objectives Primary headache disorders are common with migraine and tension headache accounting for the vast majority of cases. A smaller proportion suffer from trigeminal autonomic cephalgia (TAC). We present a 23-year-old Caucasian female who described characteristic, episodic headaches starting with a dull retro-orbital/bi-frontal pressure evolving, over the course of 1 minute, to experience florid periorbital ecchymosis. While this phenomenon has been described in the literature, the characteristics of our case are unique and noteworthy of reporting. 